\title{
Erratum to: A complete genome assembly of Glaciecola mesophila sp. nov. sequenced by using BIGIS-4 sequencer system
}

\author{
YUAN LiNa $^{1 \dagger}$, REN LuFeng $^{1 \dagger}$, LI YunTao $^{2}$, HAN WeiJing ${ }^{2}$, YU Yong $^{3}$, CHU YaNan $^{1}$, \\ LIU GuiMing ${ }^{1}$, YU Dan ${ }^{1}$, TENG MingJing ${ }^{1}$, WANG Liang ${ }^{1}$, WANG XuMin ${ }^{1}$, \\ ZHOU XiaoGuang ${ }^{2}$, YU YuDe ${ }^{2 *} \&$ YU Jun ${ }^{1 *}$ \\ ${ }^{1}$ Key Laboratory of Genome Sciences and Information, Beijing Institute of Genomics, Chinese Academy of Sciences, Beijing 100029, China, \\ ${ }^{2}$ Institute of Semiconductor, Chinese Academy of Sciences, Beijing 100083, China; \\ ${ }^{3}$ SOA Key Laboratory for Polar Science, Polar Research Institute of China, Shanghai 200136, China
}

Erratum to: SCIENCE CHINA Life Sciences, September 2011 Vol.54 No.9: 835-840 doi: 10.1007/s11427-011-4211-9

Figure 4 in the original version is incorrect. The correct figure is as follows:


The online version of the original article can be found at http://dx.doi.org/10.1007/s11427-011-4211-9 